\title{
Normalization of Multifocal Acoustic Radiation Force Impulse Images
}

\author{
Nuria GONZÁLEZ-SALIDO, Jorge CAMACHO \\ Ultrasonic Systems and Technology Group (GSTU) \\ Spanish National Research Council (CSIC) \\ Serrano 144, 28006 Madrid, Spain; e-mail: nuria.g.s@csic.es \\ (received July 8, 2016; accepted February 8, 2017)
}

\begin{abstract}
Imaging the tissue displacements caused by Acoustic Radiation Force Impulse (ARFI) provides qualitative tissue elasticity maps around the focus. To increase imaging range, multi-focus techniques combine several images obtained with different focal depths. Since the acoustic radiation force depends on focus depth, axial distance and steering angle, a normalization process is required before blending multi-focal ARFI images so that changes in the displayed displacements represent true tissue elasticity variations. This work analyzes the sources of displacement variability in multi-focal-zone ARFI and proposes a procedure to normalize and combine partial images. The proposal is based on the system focal configuration, transducer characteristics and global tissue parameters found by ultrasonic measurements. Performance of the proposed algorithm is experimentally evaluated with tissue mimicking phantoms.
\end{abstract}

Keywords: elastography; radiation force; ARFI; multifocal ARFI.

\section{Introduction}

The biomechanical properties of tissues are linked to function and pathology but cannot be directly assessed with conventional imaging techniques. However, using some kind of mechanical stimulus, it is possible to detect hardness heterogeneity within an imaged region. In particular, the detection of stiff nodes surrounded by soft tissue is of interest for the diagnosis of several diseases, including tumors, for which some ultrasonic techniques have been developed (PARKER et al., 2005).

Static compression of the tissue with an ultrasonic array was used in a pioneering work, where the gradient of displacements between pre and post compression A-scans gave a relative strain image (OPHIR et al., 1991). Other external means have been also proposed for tissue stimulation, such as mechanical shakers (GAO et al., 1995) or vibrators for transient elastography (CATHELIne et al., 1999).

The possibility of mechanically stimulate the tissue with Acoustic Radiation Force (ARF) was also demonstrated (Sugimoto et al., 1990). ARF is produced by the transfer of momentum from the ultrasonic wave to an attenuating medium, where the pressure and particle velocity become out of phase. Acoustic Radiation
Force Impulse (ARFI) produces a transient force $\mathbf{F}$ by means of a high-intensity focused pushing beam, whose magnitude is given by (STARRITT et al., 1991):

$$
|\mathbf{F}|=\frac{2 \alpha I}{c},
$$

where $\mathbf{F}$ points toward the sound propagation direction, $\alpha$ is the absorption coefficient, $I$ the timeaveraged beam intensity and $c$ is the longitudinal wave speed of sound. In soft tissue, absorption dominates scattering and $\alpha$ is the attenuation coefficient. This force causes small tissue displacements, typically below $10 \mu \mathrm{m}$, that are commonly estimated by crosscorrelation techniques (VARGHESE, OPHIR, 1996), zero crossing tracking (SRINIVASAN, OPHIR, 2005) or spectral shift estimation (HoYT et al., 2006).

In linear conditions, following the Hooke's law, displacements are proportional to force and inversely proportional to tissue stiffness. Therefore, measured tissue displacements produce a strain map that differentiates soft from hard regions (NightingaLe, 2011), although they do not provide quantitative elasticity values.

The sequence to obtain a Single Focus Image (SFI) in ARFI begins with a tracking beam to get a reference A-scan (Fig. 1). A tracking beam is a conventional pulse-echo A-scan acquisition. Then, a focused 


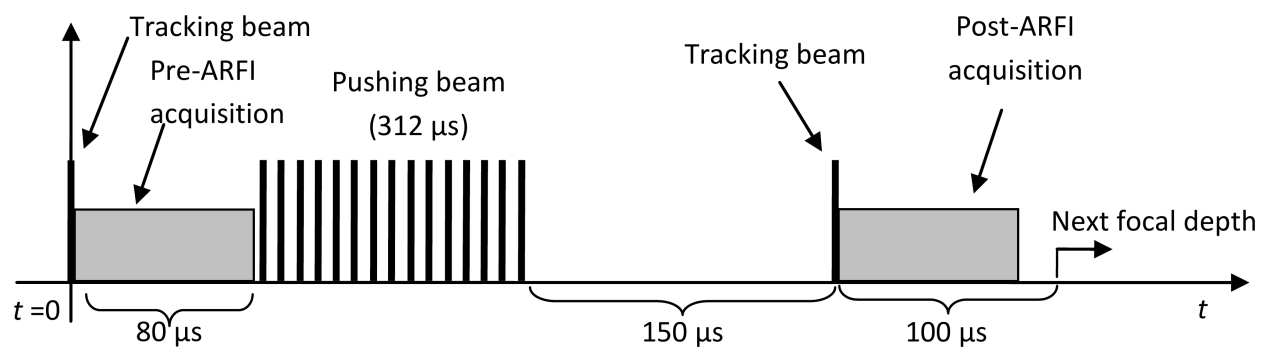

Fig. 1. ARFI sequence with some parameter values used in the experiments.

high energy pushing beam is emitted for acoustic radiation force generation, usually with a burst excitation. The high acoustic intensity at focus produces tissue displacements, but no data is recorded at this stage. A final tracking beam acquires the post-pushing A-scan with tissue displacements, which are obtained by cross correlation between pre- and post-pushing A-scans. The process is repeated in a line per line basis to get the whole linear or sector scan ARFI image.

The Region of Excitation (ROE), where measurable displacements are produced, has an extent essentially limited to the focal length. In Super-sonic Shear Imaging or SSI multiple pushing foci are produced along a line at a rate such that they move faster than the shear wave propagation velocity. This forms a supersonic mach cone that produce tissue displacements and low-speed shear waves at both sides of the propagation path. The transverse elasticity modulus is obtained from the propagation velocity of the generated shear waves (SARVAZYAN et al., 2011; BERCOFF et al., 2004; MontALDo et al., 2009), although ultrafast imaging equipment ( $>1000$ frames per second) is required to track their propagation (TÄNTER, FINK, 2014).

With a similar concept, several single focus ARFI excitations can be applied at selected intervals in the propagation direction to increase the ROE (SHARMA et al., 2005). Ultrafast acquisition hardware is not required here as long as no shear wave tracking is performed. Figure 2 shows schematically the concept of multi-focal ARFI imaging for linear and sector scans. In both cases a large ROE is achieved by combining SFIs obtained with different focus depths into a single image. Also, several pushing pulses can be sequentially sent before the common post-pushing tracking beam to improve response time (RosENZWEIG et al., 2015).

Anyway, generated ARF vary in every ROE and among them due to changes in beam intensity and shape with depth, focus position and steering angle. These variations will produce differences on measured displacements that are not directly related with tissue elasticity differences. Some procedure is required to decouple these effects from true tissue stiffness variations, one of the main objectives of the present work.

Although we have previously used the average of displacements to compensate the angular dependence

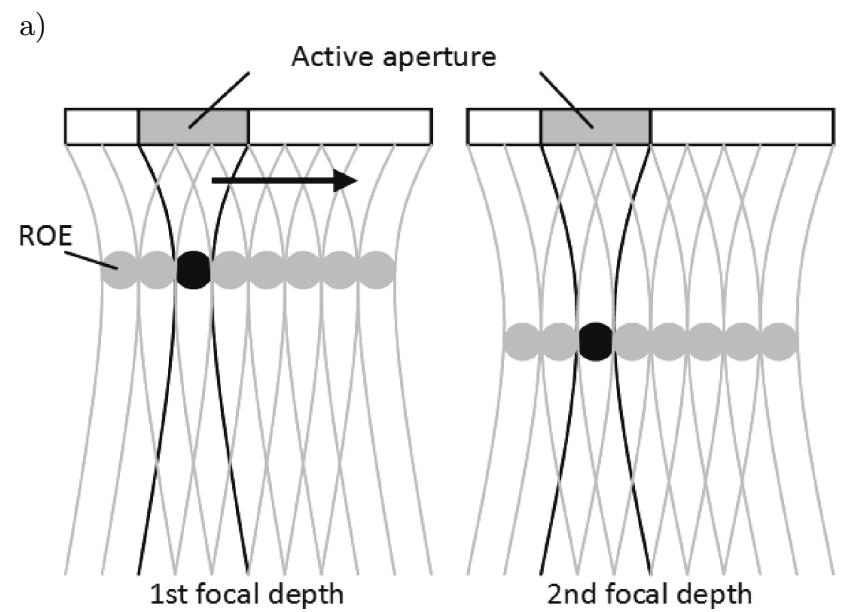

b)

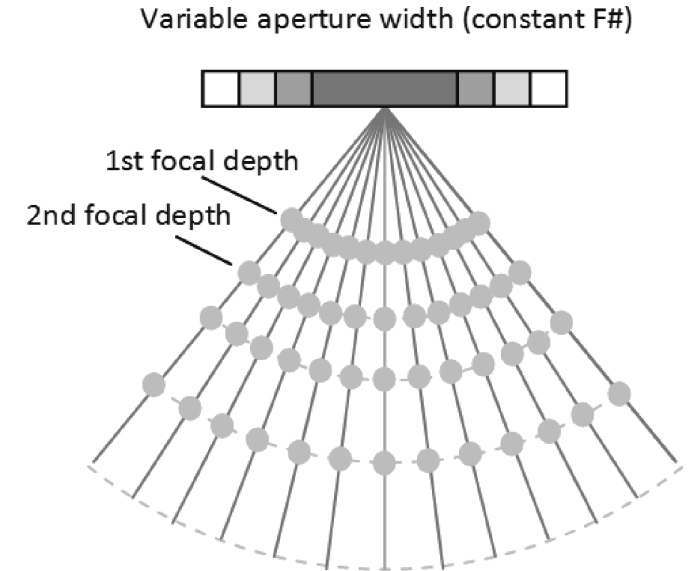

Fig. 2. Multi-focal zone ARFI imaging concept: a) linear scan b) sector scan.

of ARF (GoNzÁLEZ-SALIDo et al., 2015), we consider that measured displacements should not intervene in the equalization process to avoid biasing the results, unless those measured in regions of known homogeneous elasticity (i.e. phantoms). If, for example, data used to obtain the compensating functions contain a small hard inclusion, the equalization process would tend to hide its presence.

On the other hand, sector images are used in this work to increase the field of view in the lateral direction. This is an important aspect for full angle spatial compound imaging for breast cancer detection 
(CAMACHO et al., 2012; OpIELIŃsKi et al., 2013; 2014), were ARFI could be included as a complementary image modality to display tissue stiffness variations.

The proposed SFI equalization technique is based on the focal configuration, array characteristics and global tissue parameters that can be estimated from B-scan images, like global attenuation coefficient. Foci positions and length of focal zones are obtained for a given image range. Sources of displacement variability not directly related to tissue elasticity are identified and compensated, while using measured displacements is avoided to better reflect real elasticity differences in the final image.

\section{Proposed methodology}

Ideally, a homogeneous elasticity medium should provide a uniform displacement image with the same color for all pixels regardless the transmit configuration (focus depth, number of SFIs, etc.). However, as it was mentioned, changes in the beam intensity with depth, focus position and steering angle, produce force and displacements differences unrelated to tissue elasticity.

Each one of these sources of variations is modeled to find compensating functions that equalize the displacements and to reflect, as much as possible, the true elasticity variations of real tissue. The proposed process for multi-focal ARFI image compounding equalization is shown in Fig. 3.

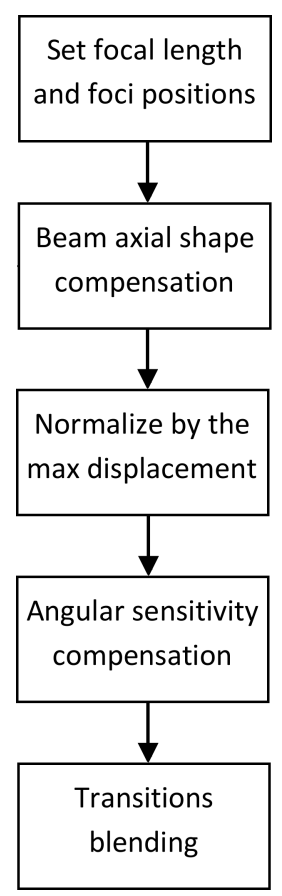

Fig. 3. Process to combine several single focused ARFI images.

First, foci positions are obtained for a given $F / \#=$ $z_{0} / D$, where $z_{0}$ is focal depth, $D$ is the active aperture size, $D=N \cdot d, N$ is the number of elements and $d$ is the element pitch. $F / \#$ is kept constant in all SFIs to get a constant beam width at focus, so that $N$ changes as a function of focal depth:

$$
N\left(z_{0}\right)=\frac{z_{0}}{d \cdot F / \#}
$$

Beam axial intensity around the focus position is taken into account to compensate the corresponding force and displacement variations in every ROE. Then, normalization by the maximum displacement compensates the intensity variations due to attenuation effects: acoustic radiation force increases linearly with attenuation, beam intensity decreases exponentially with attenuation and range and aperture size increases with depth to keep $F / \#$ constant. Angular sensitivity compensation equalizes the varying response of the transducer elements with steering angle. Finally, a transition blending procedure reduces the striped aspect and smooths the final image.

\subsection{Foci positions}

In practice, measurable ARFI displacements are only generated within the ROE, whose length can be considered the focal length at $-6 \mathrm{~dB}$. For linear arrays (KINO, 1987):

$$
L=7.1 \lambda(F / \#)^{2}
$$

Lower $F$ /\# focused beams use more active elements, deposit larger amounts of energy and generate greater displacements in a smaller region around the focus. These are easier to measure at expenses of a smaller ROE. As a trade off among these factors a constant $F / \#$ between 1 and 3 is frequently selected for ARFI.

If $F / \#$ is kept constant, the minimum number of focal zones is the ratio of the image range to the focal length $L$. For the scan configuration of the experimental work defined in Table 1, the focal length is $L=10.8 \mathrm{~mm}$ and, for a $50 \mathrm{~mm}$ depth image, 5 focal zones are required. These are set at focal depths $z_{o}=10,20,30,40$ and $50 \mathrm{~mm}$.

Table 1. Parameters of multi-focal ARFI.

\begin{tabular}{|c|c|c|c|c|}
\hline $\begin{array}{c}c \\
{[\mathrm{~m} / \mathrm{s}]}\end{array}$ & $F / \#$ & $\begin{array}{c}z_{0} \\
{[\mathrm{~mm}]}\end{array}$ & $\begin{array}{c}f \\
{[\mathrm{MHz}]}\end{array}$ & $\begin{array}{c}d \\
{[\mathrm{~mm}]}\end{array}$ \\
\hline 1540 & 1.8 & $10,20,30,40$ and 50 & 3.2 & 0.22 \\
\hline
\end{tabular}

\subsection{Beam intensity as a function of depth}

Displacement spatial distribution is affected by the intensity field $I$, which depends on depth and steering angle. Although in previous work we computed the depth-dependent profile as the mean displacement over all lateral locations in a homogeneous elasticity area (GONZÁLEZ-SALIDO et al., 2015), this requires careful 
selection of such region to avoid biasing the results. Instead, here we propose separating axial and angular dependences that are a priori known as functions of transducer characteristics only.

Beam intensity in the axial direction for a transducer is given by (KINO, 1987),

$$
I(z)=\left(\left[\left(\frac{z_{0}}{z}\right) \operatorname{sinc}\left(\frac{1}{2 S}\right)\left(\frac{z_{0}}{z-1}\right)\right]\right)^{2}
$$

where $S=z_{0} \lambda /(D / 2)^{2}$. For focused transducers with $S \ll 1$ it is simplified to:

$$
\begin{aligned}
I(z) \approx\left(\operatorname{sinc} \frac{z-z_{0}}{2 S z_{0}}\right)^{2} & =\left(\operatorname{sinc} \frac{z-z_{0}}{2 z_{0}^{2} \lambda /(D / 2)^{2}}\right)^{2} \\
& =\left(\operatorname{sinc} \frac{z-z_{0}}{2 \lambda /(F / \#)^{2}}\right)^{2} .
\end{aligned}
$$

Equation (5) represents the axial beam intensity in the proximities of the focus. It is independent of displacements and, hence, of tissue stiffness. Thus, it can be used to equalize acoustic intensity axial variations around the focus in every ROE.

\subsection{Normalization of maximum displacement}

If $F / \#$ is kept constant, the number of active elements of the array increases linearly with $z_{o}$ (Eq. (2)) and intensity grows accordingly because of larger radiation area. On the other hand, tissue attenuation reduces the beam intensity exponentially with depth. If $I_{E}$ is the intensity due to a single array element and $\alpha$ is the attenuation coefficient at the transducer frequency,

$$
I=N I_{E} e^{-2 \alpha z_{0}}=\frac{z_{0} I_{E}}{d(F / \#)} e^{-2 \alpha z_{0}} .
$$

Substitution in Eq. (1) yields:

$$
F\left(z_{0}\right)=\frac{2 \alpha z_{0} I_{E}}{(F / \#) c d} e^{-2 \alpha z_{0}}=2 k \alpha z_{0} e^{-2 \alpha z_{0}}
$$

with $k=I_{E} /(F / \#) c d$. The maximum of $F$ is found at:

$$
z_{0 \max }=\frac{1}{2 \alpha},
$$

where $\alpha$ should be expressed in linear scale $\left[\mathrm{m}^{-1}\right]$.

Figure 4 shows the normalized force at focus for the parameters of Table 1 as a function of the focus position for several attenuation coefficients expressed in the more usual form of $\mathrm{dB} / \mathrm{cm}$ for the actual frequency. Force initially grows due to larger apertures that keep $F$ /\# constant but, at certain depth, the amplitude starts to decrease because the exponential term corresponding to tissue attenuation prevails. The maximum is found at range $z_{0 \text { max }}$ given by Eq. (8).

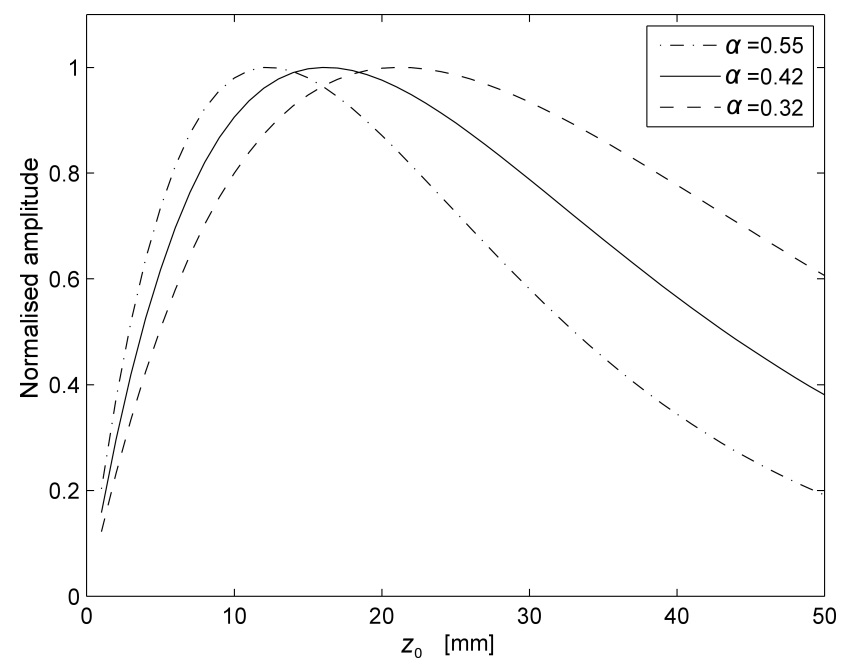

Fig. 4. Normalized force versus focus position for the parameters of Table 1.

Figure 5 shows the axial profiles of the displacements given by Eq. (7) with $I_{E}$ computed by Eq. (4) for a single element with $D=d$, normalized to the maximum force at $z_{0 \max }$ for the 5 defined focal zones and with $\alpha=1.34 \mathrm{~dB} / \mathrm{cm}$ at the transducer frequency $(\alpha=0.42 \mathrm{~dB} / \mathrm{cm} / \mathrm{MHz})$. These graphs reveal that both, the beam-shape within each SFI and the maximum displacement at each focus position, must be considered in the equalization process. Furthermore, it is essential that displacement measurements be made immediately after removing the pushing beam and before shear waves deform the displacement field.

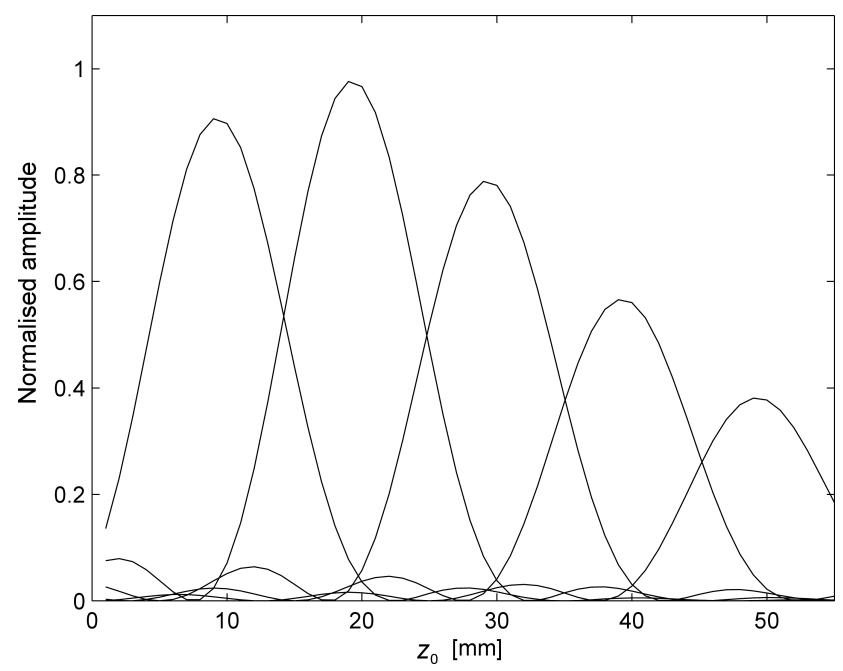

Fig. 5. Normalized displacement versus focus position predicted by (7) for configuration in Table 1.

\subsection{Angular sensitivity}

Sector scan images offer a broader field-of-view. However, angular sensitivity of the transducer de- 
creases with steering angle due to diffraction caused by the finite width of elements. The beam intensity depends on steering angle and these variations must be compensated for ARFI imaging.

According to (KINO, 1987), the theoretical angular dependence of intensity at constant distance $z_{0}$ for a rectangular element of width $w_{e}$ is:

$$
I(\theta)=I_{0}\left(\frac{w_{e}}{\sqrt{\lambda z_{0}}} \operatorname{sinc}\left(\frac{w_{e}}{\lambda} \sin \theta\right) \cos \theta\right)^{2 \cdot k},
$$

where $\theta$ is the steering angle and $k$ is an empirical factor to adjust the theoretical response with the actual lateral array response. This can be obtained by measuring the actual array lateral pattern with a pointlike reflector in water immersion. A least squares fit technique provides the value of $k(k=1.11$ in our experiments). Divergence from the ideal value $k=1$ can be due to multiple reasons, being mechanical coupling between elements the most common.

To equalize the lateral response, the amplitude of each A-scan in a sector SFI is divided by the nominal intensity at the actual steering angle. Figure 6 shows Eq. (9) for the nominal parameters of Table 1 and different element widths.

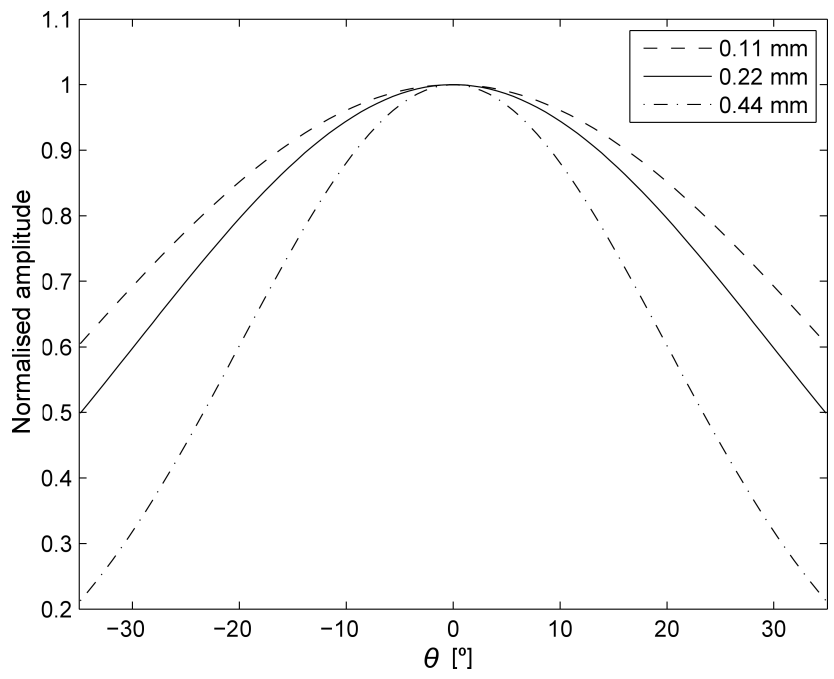

Fig. 6. Angular sensitivity for elements widths $w_{e}=0.11$, 0.22 and $0.44 \mathrm{~mm}$.

\subsection{Transitions blending}

The steps above should compensate most of the causes of variations in measured displacements: beam shape around focus within every SFI, axial beam intensity changes as a function of focus position and angular dependence of array sensitivity. In principle, these should be sufficient to obtain a compensated multifocal image. However, tissue response is affected by inertia and shear wave propagation that yield slight deviations in the displacements measured in adjacent SFI images. Such deviations produce a striped aspect of the image after compounding. To correct this artifact, a transition blending algorithm is used.

Each SFI is divided in three regions: a central area where no correction is required and two transition bands where adjacent images overlap. On each image, the upper transition band is multiplied by a linear windowing function going from 0 to 1 , and the bottom transition band by a similar window going from 1 to 0 . Then, SFIs are added together to obtain the multi-focal-zone image. The blending scheme is shown in Fig. 7 for the parameters in Table 1 and $5 \mathrm{~mm}$ overlap among windows.

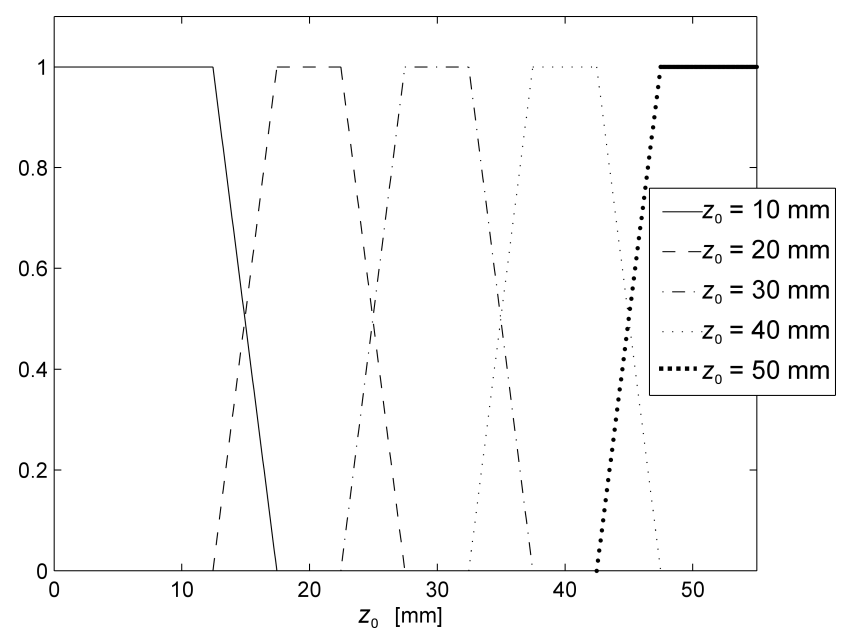

Fig. 7. Transition blending coefficients for parameters of Table 1 and $5 \mathrm{~mm}$ overlap.

\section{Experimental verification}

Experiments were carried out using a Sitau-112 phased array system (Dasel SL, Spain) with 128 parallel pulse-echo channels and a $3.2 \mathrm{MHz}, 128$-elements, $0.22 \mathrm{~mm}$ pitch array (model nr. P2-4/30EP, Prosonic, Korea). For ultrasonic data and ARFI processing, custom scripts were implemented in Matlab (The Mathworks Inc, USA).

We used two homemade homogeneous phantoms with different elasticity and a commercial medical breast tissue phantom (Blue Phantom, USA, $c=$ $1440 \mathrm{~m} / \mathrm{s}, \alpha=0.32 \mathrm{~dB} / \mathrm{cm} / \mathrm{MHz}$ ), that includes structures mimicking hard masses and cysts. The homogeneous phantoms were made from gelatin, graphite, isopropanol, water, and formalin (25\% of formaldehyde) following the procedure described in (BILGEN, 1997). The proportions were adjusted to get the elasticity modules listed in Table 2 . The ratio between graphite, gelatin and isopropanol was kept constant to achieve a nominal attenuation $\alpha=0.42 \mathrm{~dB} / \mathrm{cm} / \mathrm{MHz}$ and a sound speed $c=1542 \mathrm{~m} / \mathrm{s}$.

A script was developed to automatically generate $70^{\circ}$ sector SFIs with scan lines at angular steps of $0.75^{\circ}$ 
Table 2. Composition and measured elasticity of each phantom.

\begin{tabular}{|c|c|c|c|c|c|c|}
\hline Phantom & $\begin{array}{c}\text { Gelatin } \\
{[\mathrm{gr}]}\end{array}$ & $\begin{array}{c}\text { Water } \\
{[\mathrm{l}]}\end{array}$ & $\begin{array}{c}\text { Isopropanol } \\
{[\mathrm{ml}]}\end{array}$ & $\begin{array}{c}\text { Graphite } \\
{[\mathrm{gr}]}\end{array}$ & $\begin{array}{c}\text { Formalin } \\
{[\mathrm{ml}]}\end{array}$ & $\begin{array}{c}\text { Target E } \\
{[\mathrm{Kpa}]}\end{array}$ \\
\hline $\mathrm{A}$ & 96 & 1.2 & 96.25 & 117.6 & 3.6 & 25 \\
\hline $\mathrm{B}$ & 118.2 & 1.2 & 96.25 & 117.6 & 3.6 & 40 \\
\hline
\end{tabular}

and focus depths of pushing beams ranging from 10 to $50 \mathrm{~mm}$ at $10 \mathrm{~mm}$ interval, as given in Table 1 . The number of active elements of the array for each SFI was calculated from Eq. (2) to keep $F / \#=1.8$, giving a maximum image depth of $60 \mathrm{~mm}$ with the full-size aperture $D=128 \times 0.22=28.16 \mathrm{~mm}$.

Acquisitions were performed by coupling the array to the phantom with acoustic gel. The pushing beam duration was set to $312 \mu \mathrm{s}$ and the post-tracking beam was fired $150 \mu$ s after removing the pushing pulse. RF data from each excitation location were acquired at $40 \mathrm{MHz}$, up-sampled by a factor of 32 and processed offline using a moving 1D-cross-correlation algorithm to estimate axial displacements. Correlation parameters were $4 \lambda$ kernel with $0.8 \mathrm{~mm}$ overlap and $0.05 \mathrm{~mm}$ of maximum lag. For each phantom, attenuation was estimated by linear regression in $z$ (depth) of a logcompressed B-Scan image obtained with plane-wave emission (no focus).

\subsection{Phantom $A$}

Figure 8 shows the unprocessed ARFI image of phantom A. Five bands corresponding with focus positions are clearly seen and the effects of angular sensitivity, maximum displacement and beam axial shape variations can be appreciated. Despite the phantom elasticity is homogeneous, the image is not, showing bands, angular and axial gradients.

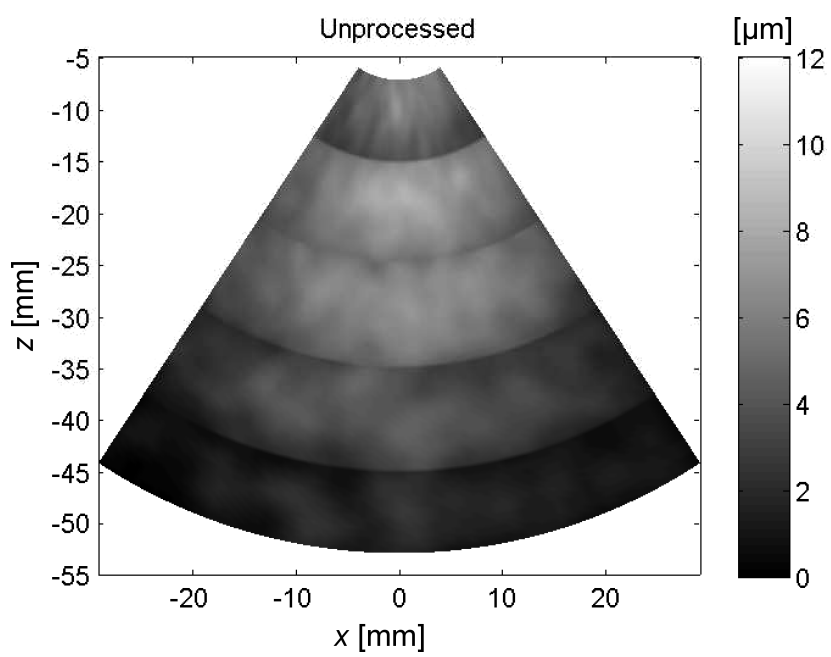

Fig. 8. ARFI image of phantom A without normalization.

Figure 9a shows the peak displacements at each focus position at $0^{\circ}$ steering (asterisks) together with a)

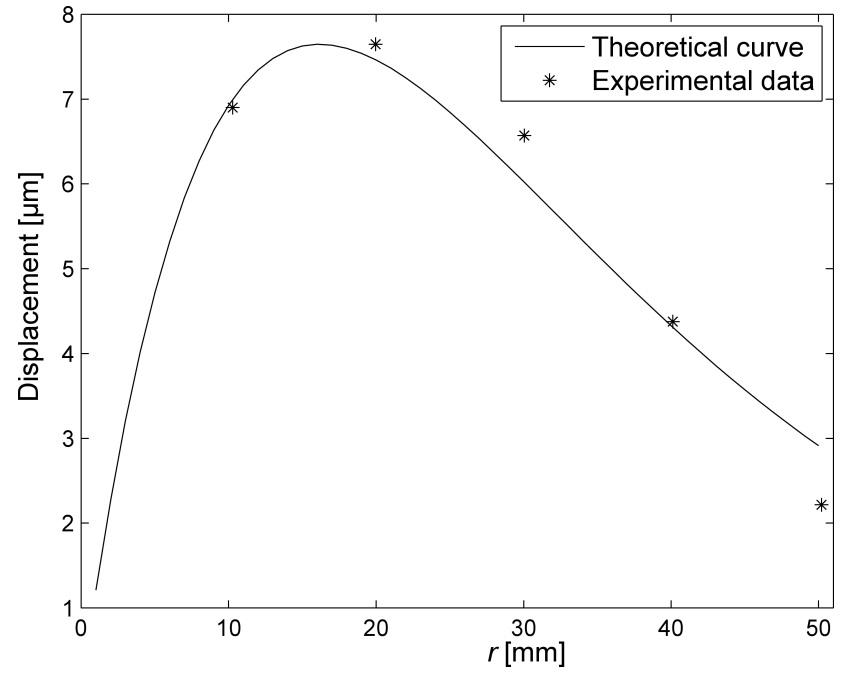

b)

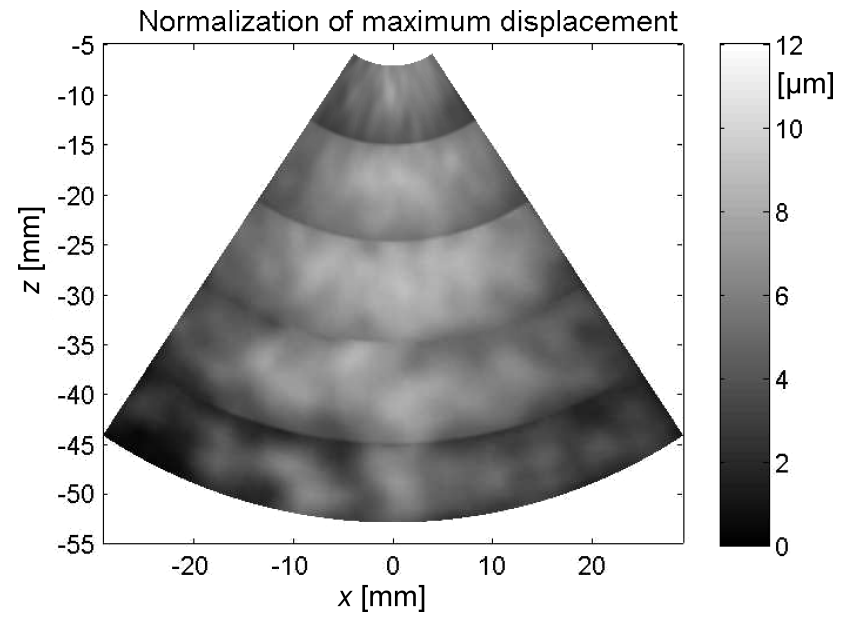

Fig. 9. a) Maximum displacement at each SFI (asterisks) and theoretical value (solid line) scaled to measured data; b) ARFI image after maximum displacement correction.

theoretical values predicted by Eq. (7) (solid line), scaled to fit experimental data in $\mu \mathrm{m}$. This scaling is only intended to graphically compare both magnitudes and it was not used for equalization, which is performed with normalized values only. Figure $9 \mathrm{~b}$ shows the ARFI image obtained after axial equalization with normalized theoretical values given by Eq. (7). As expected, displacement variability with depth is reduced, although not completely removed.

Figure 10a shows, with asterisks, displacements against steering angle measured at $30 \mathrm{~mm}$ depth 
a)

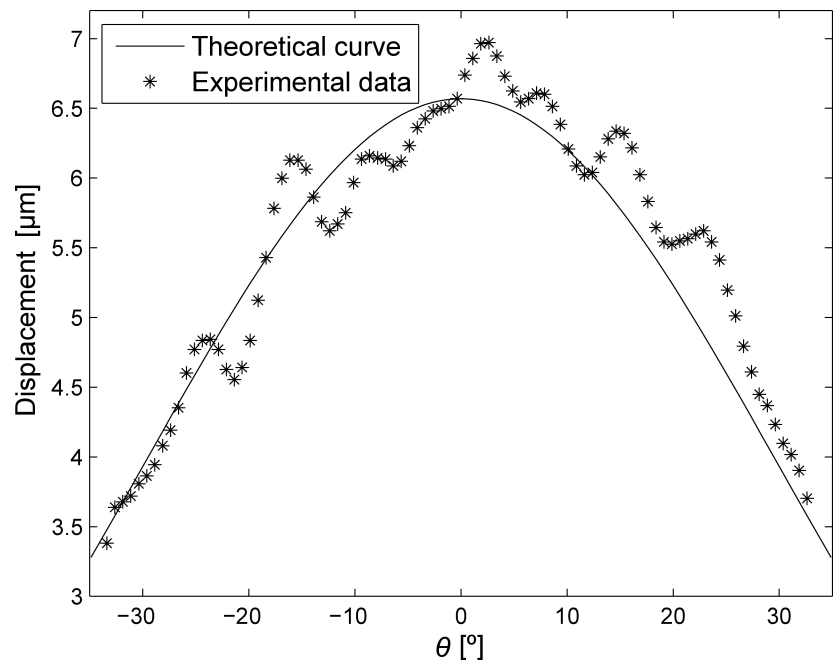

b)

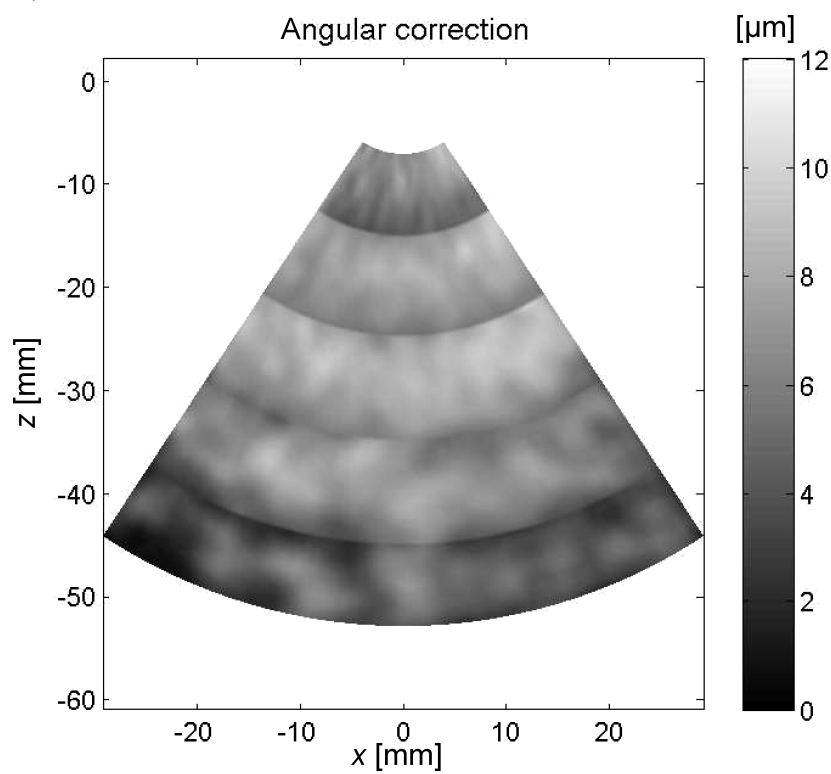

Fig. 10. a) Measured displacement at $30 \mathrm{~mm}$ against steering angle for experimental data (asterisks) and according to (7) (solid line) scaled to measured data; b) ARFI image after angular sensitivity correction.

(the focal position of the third SFI). Experimental data reasonably agrees with lateral sensitivity predicted by Eq. (9) (solid line), also scaled to measured data. Figure $10 \mathrm{~b}$ shows the ARFI image after angular equalization, where displacement dependence with steering angle is significantly reduced.

Figure 11a shows the axial displacement measured at the center line of the third SFI, which extends from 22.5 to $37.5 \mathrm{~mm}$ (asterisks), along with the normalized beam intensity predicted by Eq. (5) (continuous line) and scaled to measured data. Although experimental data follows the shape of a sinc function, the focal region is wider than expected. This can be ex- plained by the tissue recovery during the time elapsed from the end of excitation to displacement data acquisition, in this case $150 \mu \mathrm{s}$. The widening effect can be modeled in Eq. (5) by increasing $F / \#$ by a factor $\beta$ that, for this experiment, was estimated by mean square fit to experimental data as $\beta=1.48$ (dashed trace in Fig. 11a).

Figure 11b shows the ARFI image after equalizing the beam axial shape dependence given by Eq. (5) with $F / \#$ increased by a factor 1.48 . To this point, the three proposed corrections were applied, and a rather homogeneous image has been obtained, although SFI stripes are still seen.

a)

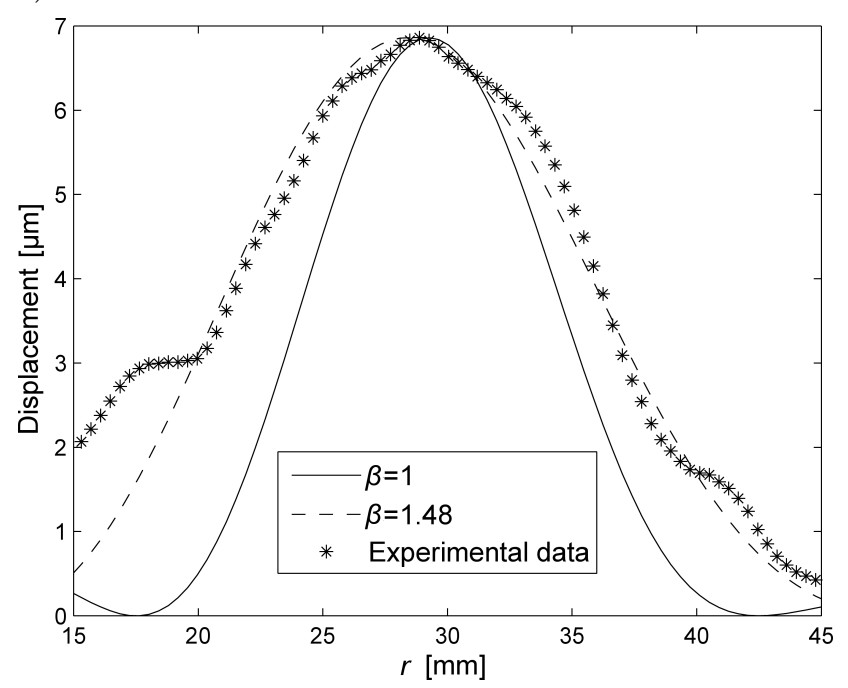

b)

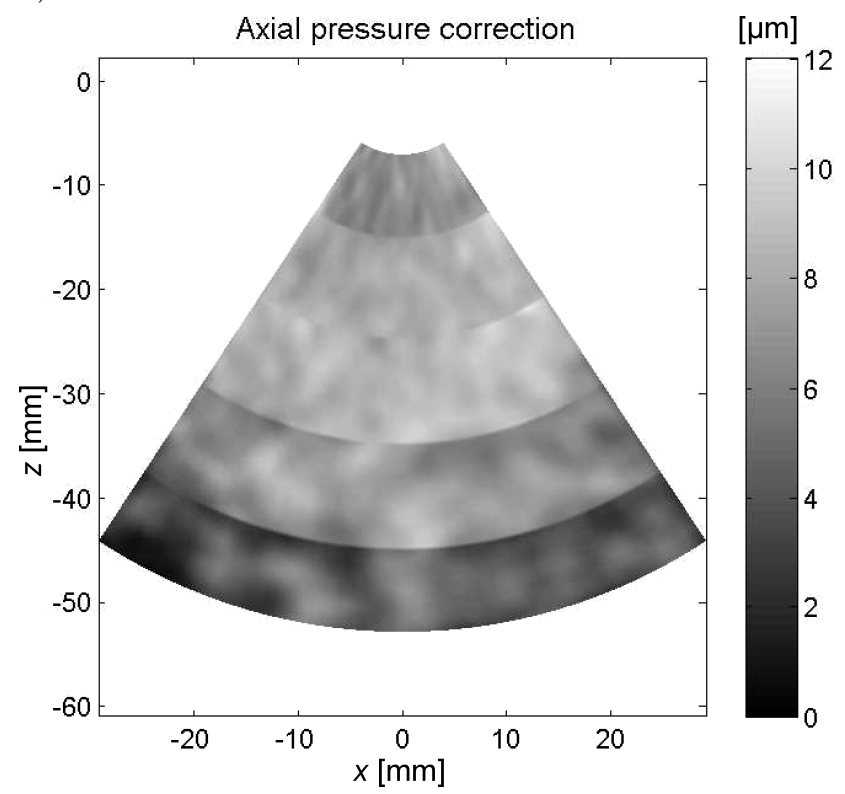

Fig. 11. a) Measured displacements versus depth for the third SFI (asterisks) along with theoretical values predicted by Eq. (4) (solid line) scaled to measured data, and the proposed correction, with $F / \#$ increased by a factor 1.48 (dashed line); b) ARFI image after beam axial shape equalization. 
a)

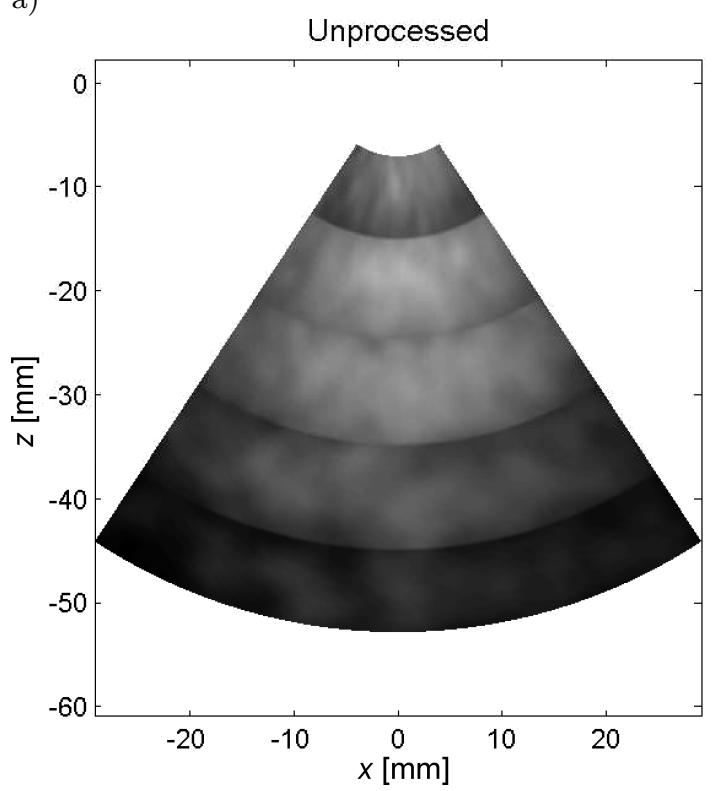

b)

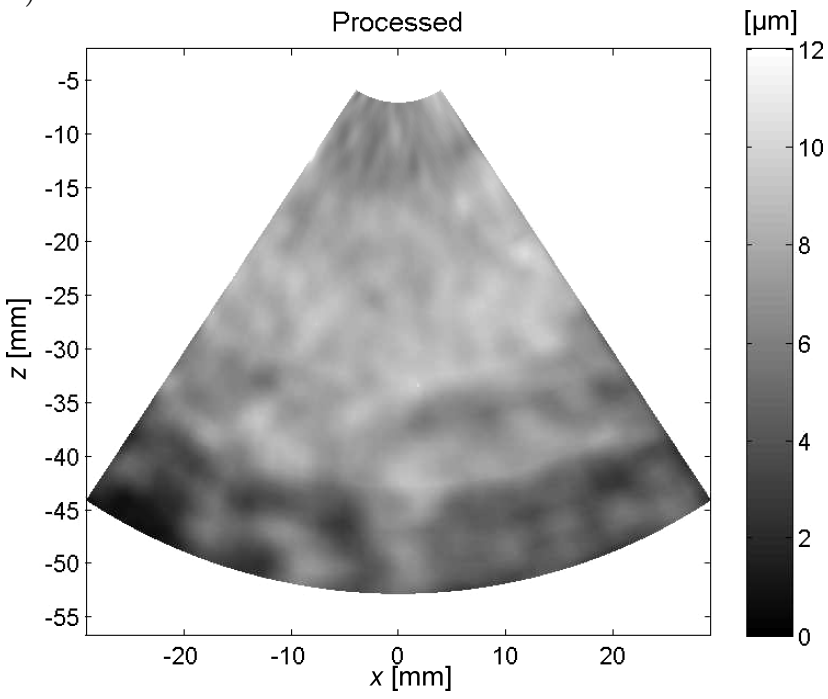

Fig. 12. Phantom A: a) Original Multifocal ARFI image, b) post-processed Multifocal ARFI image.
Figure 12a shows the original multifocal ARFI [ $\mu \mathrm{m}]$ image (top) and after correction including transition $\square^{12}$ blending (Fig. 12b). Even after processing, the last band at around $40 \mathrm{~mm}$ depth remains slightly subcompensated with regard to the image center. Original displacements within this band are below the minimum detectable level of $2.3 \mu \mathrm{m}$ under the conditions of the experiment (WALKER, TRAHEY, 1995) and the low SNR in the last band explains the lower effect of the equalization process.

To provide further numerical insight on these features, Fig. 13 shows the $0^{\circ}$ displacement profile (along the propagation axis) of the ARFI image before correction (dashed line) and after correction (solid line). The original maximum displacement variation of $8 \mu \mathrm{m}$ is reduced to $2.5 \mu \mathrm{m}$ and the standard deviation from 1.8 to $0.95 \mu \mathrm{m}$ after applying the proposed corrections in the full range up to $50 \mathrm{~mm}$.

\subsection{Phantom $B$}

Phantom B with higher elasticity modulus was imaged with the same configuration than phantom A. Figure 14 shows de Multifocal ARFI image before (top) and after (bottom) application of the proposed compensation procedure. A homogeneous image is also obtained confirming that the proposed method can be applied without previous knowledge of tissue elasticity and without using measured displacements for image normalization. The value of parameter $\beta$ was the same that used with phantom A, confirming that it is related with the delay time between the pushing and tracking beams, equal in both experiments.

The $0^{\circ}$ displacement profile for phantom B is shown in Fig. 15. Here the maximum displacement variation is reduced from to $7.5 \mu \mathrm{m}$ to $5.5 \mu \mathrm{m}$ and the standard deviation from 2.2 to $1.7 \mu \mathrm{m}$. Since phantom B is stiffer than phantom A, smaller displacements are produced, which yield lower SNR in the last two SFIs. The consequence is that the equalization process sub-

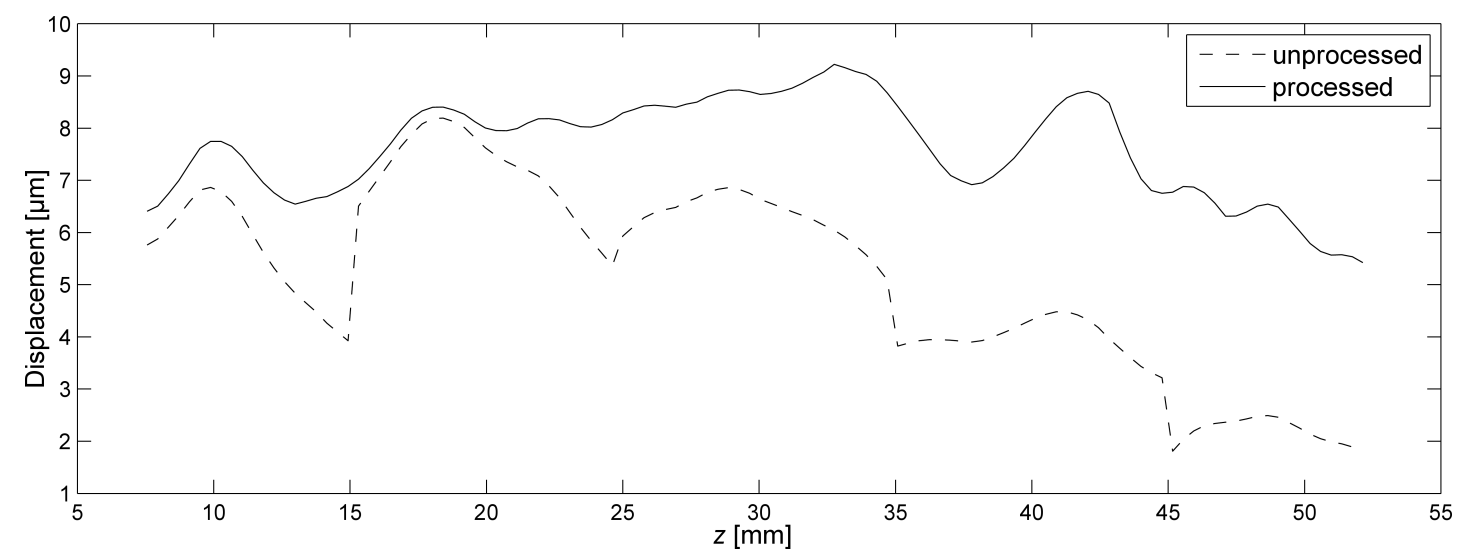

Fig. 13. Phantom A: Axial displacements measured at the center line of the ARFI image before (dashed line) and after (solid line) applying the proposed correcting actions. 
a)

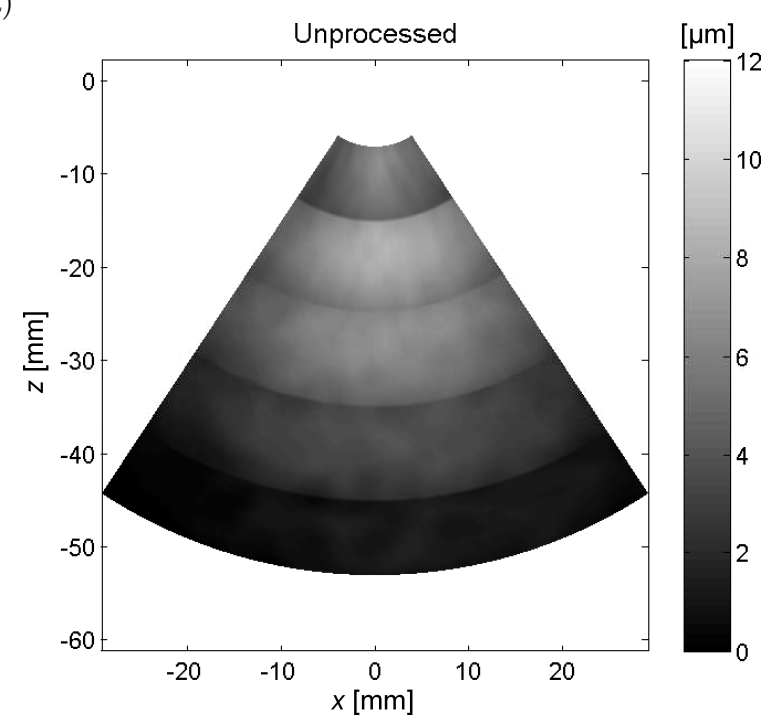

b)

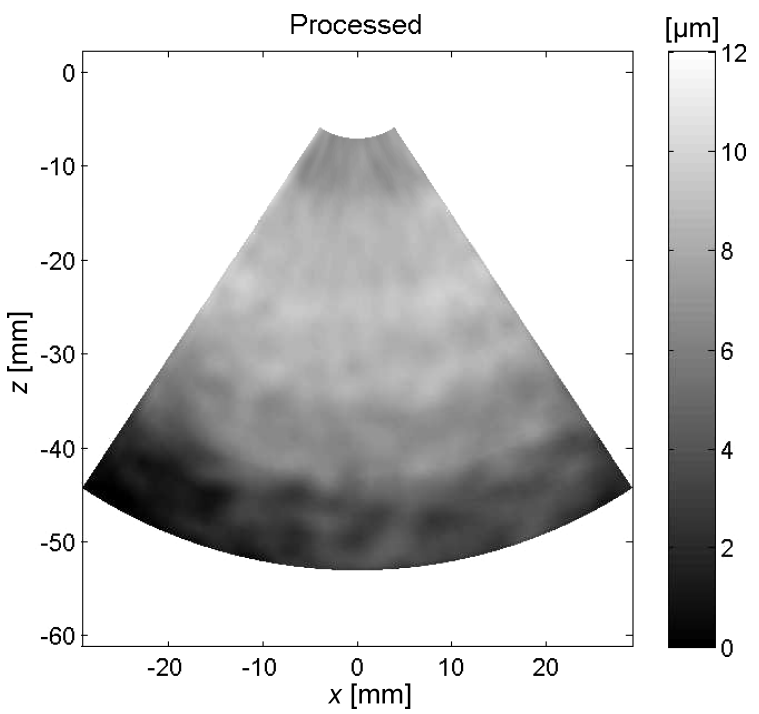

Fig. 14. Phantom B: a) original Multifocal ARFI image; b) post-processed Multifocal ARFI image. compensates image level in those bands for this phantom. The image range where the equalization is effective is reduced to about $35 \mathrm{~mm}$ in this case.

The proposed equalization technique has been proved to work with rather homogeneous phantoms without prior knowledge of their elastic properties. This way, the compensating algorithm has shown to be independent of tissue characteristics with the exception of the required estimation of attenuation.

\subsection{Breast tissue mimic phantom}

The last experiment was performed on a commercial breast phantom containing a hard mass (Blue Phantom, USA, $c=1440 \mathrm{~m} / \mathrm{s}, \quad \alpha=$ $0.32 \mathrm{~dB} / \mathrm{cm} / \mathrm{MHz})$. The same configuration than with phantoms $\mathrm{A}$ and $\mathrm{B}$ was used and no a priori knowledge of the phantom elasticity was assumed.

Figure 16a shows the conventional reflectivity B-mode sector scan image, where a $12 \mathrm{~mm}$ diameter hyperechogenic mass is seen at about $30 \mathrm{~mm}$ depth. Figure 16b shows the original multi-focus ARFI image, where transition bands, axial and angular dependence of displacements can be observed. After applying the proposed correction, a more homogeneous image is obtained (Fig. 16c), which provides cleaner stiffness information.

\section{Discussion and conclusions}

The response of multi-focal sector ARFI images presents variations due to focus position, steering angle and beam geometry at focus, aspects that are unrelated to tissue elasticity. To overcome this problem we presented a technique that combines multiple single focus ARFI images into a multifocal image performing an equalization process to avoid as much as possible the artifacts created by beam intensity variations at different depths and steering angles.

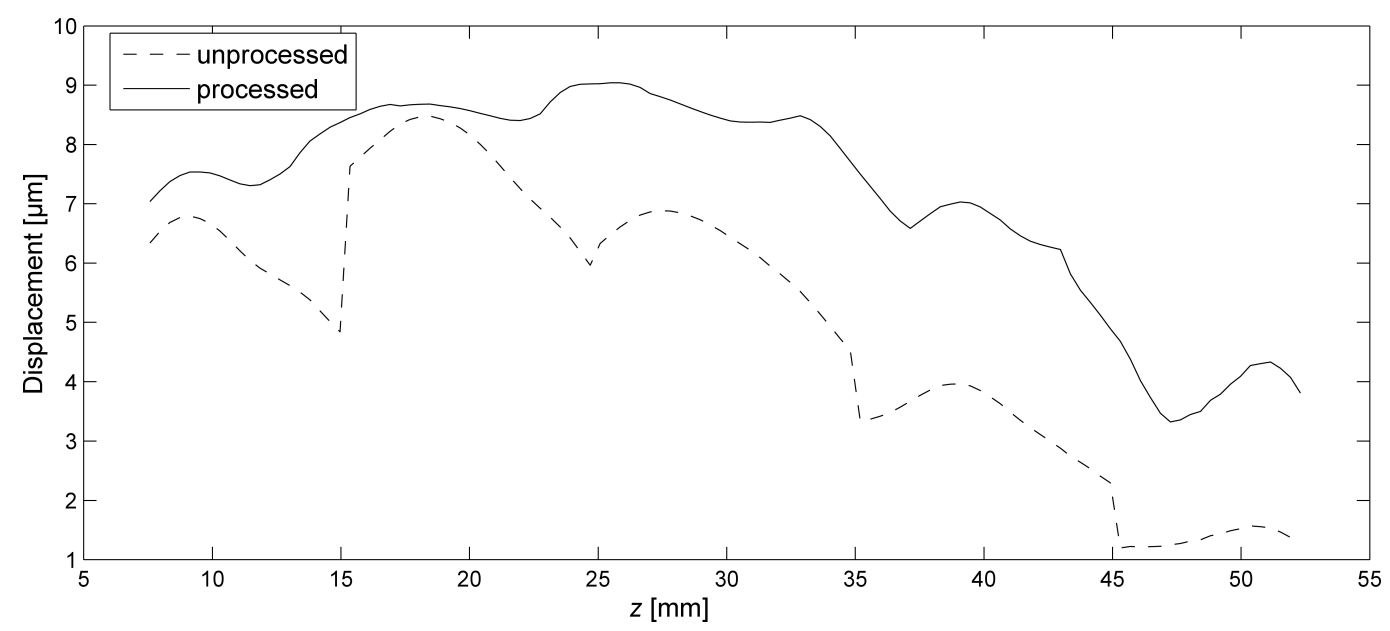

Fig. 15. Axial displacements measured at the center line of the ARFI image before (dashed line) and after (solid line) applying the proposed correcting actions. 
a)

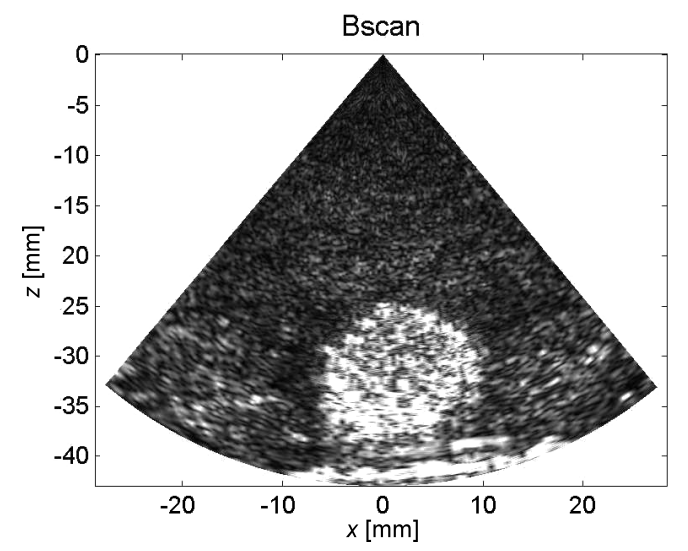

b)

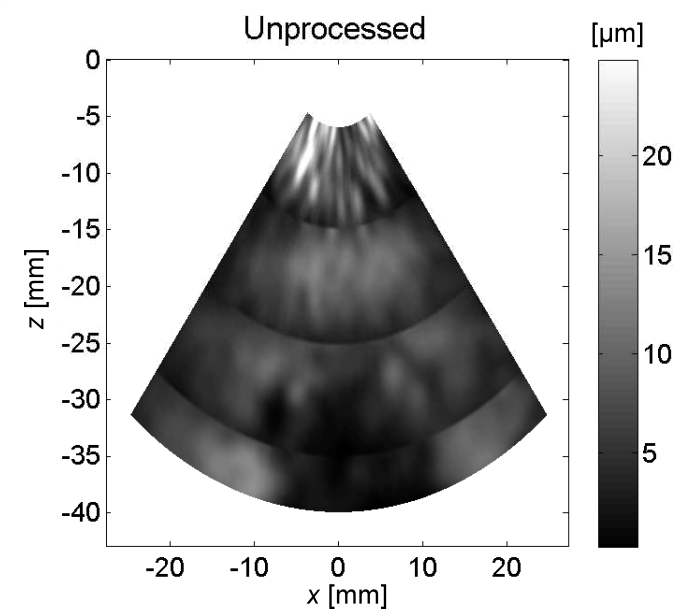

c)

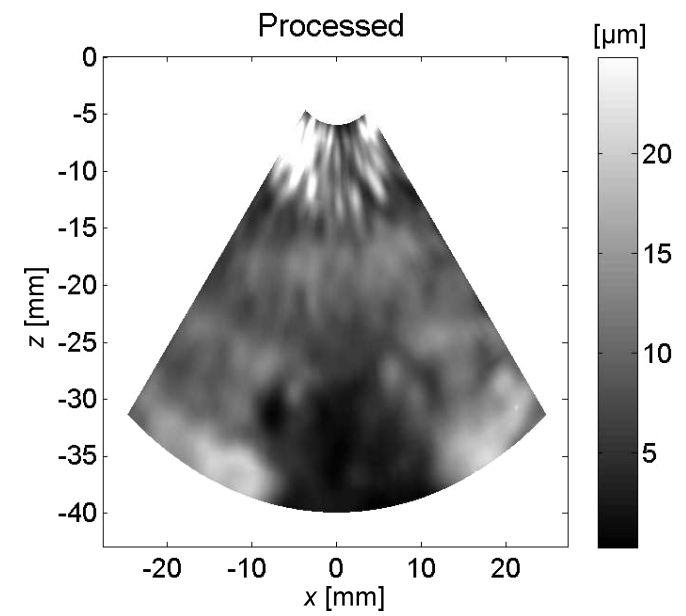

Fig. 16. Breast phantom with solid mass. a) conventional B-Scan; b) original Multifocal ARFI image; c) multifocal ARFI image after applying the proposed correction.

It has been demonstrated experimentally that the proposed procedure automatically provides an acceptable level of equalization of the final image without using displacement data, neither any a priori knowledge of the elasticity properties of the imaged tissue. This was experimentally verified with homogeneous and non-homogeneous tissue mimic phantoms.
Once the transducer array has been characterized for angular sensitivity, the only parameter that must be estimated is tissue attenuation, which was performed with ultrasonic measurements from a conventional B-mode image. In this work, attenuation was estimated by the slope of a linear regression of a logcompressed B-Scan image obtained with plane-wave emission.

In practice, the tissue attenuation coefficient is not constant and should be considered, at least, a function of depth and steering angle: $\alpha(z, \theta)$. Such function could be obtained by Ultrasound Transmission Tomography (UTT) as proposed in (WISKIN et al., 2010; OPIELIŃsKi et al., 2012; 2014; RoY et al., 2013). UTT obtains maps of attenuation and propagation velocity in the tissue, being a quite involved and computing intensive technique that operates in through-transmission. Therefore, although possible, it is not suitable for direct application with conventional equipment as is ARFI. A simpler method would estimate the attenuation along a single line from the B-scan image, perhaps after performing some moving average in $z$ and in $\theta$. This would yield an approximate map $\alpha_{E}(z, \theta)$ of the estimated attenuation coefficient in the imaged region. These points remain for future research.

Apart from this limitation, the proposed compensation technique for multifocal ARFI imaging has the advantage of depending on parameters known a priori or that can be estimated to suppress most of the effects of factors other than tissue elasticity variations on measured displacements. Furthermore, the algorithm can be applied in real-time since it is mostly based on closed formulae.

\section{Acknowledgments}

This work was jointly supported by projects DPI2013-42236-R of the Spanish Ministry of Economy and Competitivity, S2013/MIT-3024 of the Community of Madrid and by the FPU grant 12/02187 of the Spanish Ministry of Education, Culture and Sport.

\section{References}

1. Bercoff J., Tanter M., Fink M. (2004), Supersonic shear imaging: a new technique for soft tissue elasticity mapping, IEEE Transactions on Ultrasonics, Ferroelectrics, and Frequency Control, 51, 4, 396-409.

2. Camacho J., Medina L., Cruza J.F., MoreNO J.M., Fritsch C. (2012), Multimodal ultrasonic imaging for breast cancer detection, Archives of Acoustics, 37, 3, 253-260.

3. Catheline S., Thomas J.L., Wu F., Fink M.A. (1999), Diffraction field of a low frequency vibrator in soft tissues using transient elastography, IEEE Trans- 
actions on Ultrasonics, Ferroelectrics, and Frequency Control, 46, 4, 1013-1019.

4. Gao L., Parker K.J., Alam S.K., Lerner L.M. (1995), Sonoelasticity imaging: Theory and experimental verification, J. Acoust. Soc. Am., 97, 6, 3875-3886.

5. González-Salido N., Medina L., Cruza J., CamaCHO J. (2015), Implementation and Evaluation of Elastographic Techniques, Physics Procedia, 63, 97-102.

6. Hall T.J., Bilgen M., Insana M.F., KrousKOP T.A. (1997), Phantom materials for elastography, IEEE Transactions on Ultrasonics, Ferroelectrics, and Frequency Control, 44, 6, 1355-1365.

7. Hoyt K., Forsberg F., Ophir J. (2006), Comparison of shift estimation strategies in spectral elastography, Ultrasonics, 44, 99-108.

8. KINO G.S. (1987), Acoustic waves: devices, imaging and analog signal processing, Prentice Hall, Inc., A Division of Simon \& Schuster, Englewood Cliffs, New Jersey.

9. Montaldo G., Tanter M., Bercoff J., BeNECH N., FINK M. (2009), Coherent plane-wave compounding for very high frame rate ultrasonography and transient elastography, IEEE Transactions on Ultrasonics, Ferroelectrics, and Frequency Control, 56, 3, 489506.

10. Nightingale K. (2011), Acoustic Radiation Force Impulse (ARFI) Imaging: a Review, Curr. Med Imaging Rev., 7, 4, 328-339.

11. Ophir J., Céspedes I., Ponnekanti H., Yazdi Y., LI X. (1991), Elastography: A quantitative method for imaging the elasticity of biological tissues, Ultrasonic Imaging, 13, 2, 111-134.

12. Opieliński K.J. (2012), Ultrasonic Projection [in:] Ultrasonic Waves, Antunes Dos Santos Júnior [Ed.], pp. 29-58, INTECH, Rijeka, Croatia.

13. Opieliński K.J., Pruchnicki P., Gudra T., PodGórski, P., Kraśnicki T., Kurcz J., SĄsiadek M. (2013), Ultrasound Transmission Tomography Imaging of Structure of Breast Elastography Phantom Compared to US, CT and MRI, Archives of Acoustics, 38, 3, 321334 .

14. Opieliński K.J., Pruchnicki P., Gudra T., MaJEWSKI J. (2014), Conclusions from a test of multimodal ultrasound tomography research system designed for breast imaging, Forum Acusticum, 7-12 Sept., Kraków, Poland.
15. Parker K.J., Taylor L.S., Gracewski S., RuBENS D.J. (2005), A unified view of imaging the elastic properties of tissue, J. Acoust. Soc. Am., 117, 5, 27052712.

16. Rosenzweig S., Palmeri M., Nightingale K. (2015), Analysis of Rapid Multi-Focal-Zone ARFI Imaging, IEEE Transactions on Ultrasonics, Ferroelectrics, and Frequency Control, 62, 2, 280-289.

17. Roy O., Schmidt S., Li C., Allada V., West E., Kunz D., Duric N. (2013), Breast imaging using ultrasound tomography: from clinical requirements to system design, proceedings of Joint UFFC, EFTF and PFM Symposium, pp. 1174-1177.

18. Sarvazyan A., Hall T.J., Urban M.V., Fatemi M., Aglyamov S.R., Garra B.S. (2011), An overview of elastography - An emerging branch of medical imaging, Curr. Med. Imaging Rev., 7, 4, 255-282.

19. Sharma A., Trahey G., Frinkley K., Soo M.S., Palmeri M., Nightingale K. (2005), Image processing and data acquisition optimization for Acoustic Radiation Force Impulse imaging of in vivo breast masses, Proceedings of SPIE Proc. Medical Imaging, 5750, pp. 205-215.

20. SRinivasan S., OphiR J. (2003), A zero-crossing strain estimator for elastography, Ultrasound Med. Biol., 29, 2, 227-238.

21. Starritt H., Duck F., Humphrey V. (1991), Forces acting in the direction of propagation in pulsed ultrasound fields, Phys. Med. Biol., 36, 1465-1474.

22. Sugimoto T., Ueha S., Iтон K. (1990), Tissue hardness measurement using the radiation force of focused ultrasound, Proceedings of IEEE Ultrasonics Symposium, pp. 1377-1380, Honolulu.

23. TANTER M., Fink M. (2014), Ultrafast imaging in biomedical ultrasound, IEEE Transactions on Ultrasonics, Ferroelectrics, and Frequency Control, 61, 1, 102119.

24. VARghese T., Ophir J. (1996), Estimating tissue strain from signal decorrelation using the correlation coefficient, Ultrasound Med. Biol., 22, 9, 1249-1254.

25. Walker W., Trahey G. (1995), A fundamental limit on delay estimation using partially correlated speckle signals, IEEE Transactions on Ultrasonics, Ferroelectrics, and Frequency Control, 42, 2, 301-308.

26. Wiskin J., Borup D., Johnson M., Robinson D., Smith J., Chen J., PARisky Y., Klock J. (2010), Inverse scattering and refraction corrected reflection for breast cancer imaging, Proceedings of Medical Imaging, Proc. SPIE, 7629, pp. 1-12. 